\title{
Cell multiplication
}

\section{Editorial overview}

\author{
J.R. Pringle \\ Department of Biology, The University of Michigan, Ann Arbor, Michigan, USA
}

Current Opinion in Cell Biology 1990, 2:239-240

\section{Another remarkable year for cell-cycle studies}

In last year's overview, I noted what a remarkable year it had been for cell-cycle studies. Further, I argued that the stunning rate of progress was no accident, but rather a logical consequence of two factors, namely the increasing power of the available methods (particularly of genetic and recombinant DNA methods), and the increasing appreciation of the degree to which fundamental mech. anisms are conserved throughout the eukaryotic world. These arguments suggested that the rate of progress would not decline, a prediction that has been amply borne out in the event. I think that we all are indebted to the authors of the concise and penetrating reviews that follow for taking the time from their own research to pluck out for us the most important threads in the brilliant but sometimes bewildering tapestry of new results. In the meantime, the bases for last year's prediction have, if anything, been strengthened, so that I herewith repeat it with sublime confidence. In what follows, I join in the celebration of what has been accomplished, offer a few perhaps curmudgeonly caveats, and comment on some open questions and the prognosis for further progress.

\section{The increasing power of genetic and recombinant DNA approaches}

The influence of genetic approaches (and with it the use of genetically tractable organisms) has increased dramatically in all areas of cell biology (Pringle, New Biol 1990, 2:37-43), but is still perhaps most striking in cellcycle studies. In this regard, it is exciting to note that despite the venerability of classical genetics, new strategies for the identification of interesting genes continue to be developed (Pringle, 1990). Meanwhile, the power of recombinant DNA technology has been vastly increased by the development of polymerase chain reaction (PCR) methodology. The impact of PCR technology on the cellcycle field is just beginning to be felt, but it is already clear that it will facilitate enormously the identification of new members of gene families (Chang et al, Proc Natl Acad Sci USA 1990, 87:1571-1575), the identification of homologues between species (Hann et al., $J$ Cell Biol 1989, 109:3223-3230), and the sequencing of mutant alleles of interesting genes (Landis et al, Nature 1989, 340:692-696). Among other things, PCR technology will surely help to resolve the residual questions as to the degree of generality of cell-cycle mechanisms (see below).

\section{The conservation of fundamental mechanisms}

The past year has brought yet more confirmation of the conservation of fundamental cell-cycle mechanisms. Thus, Futcher (pp 246-251), in reviewing the yeast cell cycle, freely intermingles results obtained with clam, starfish, fy, frog, and human cells, while most of the other reviews that follow incorporate (directly or indirectly) results obtained with the genetically tractable yeasts. Morris (pp 252-257) points out some striking additional examples of protein similarities between fungal and animal cells (e.g. the Aspergillus bimG product, $86 \%$ identical to mammalian phosphoprotein phosphatase 1). He also points out that some fascinating fungal genes (nimA, bimE, $R A D 9$ ) are not yet known to have mammalian homologues. Given the recent history of the field, however, it would be rash to predict that such homologues will not be found. Meanwhile, evidence has appeared suggesting that the conserved apparanus of mitodic control is also present in algae and higher plants (John et al, Plant Cell 1989, 1:1185-1193). More problematic is the situation in prokaryotes. Lutkenhaus (pp 241-245) very nicely describes the great progress being made in understanding the temporal and spatial control of DNA replication, nucleoid segregation, and septation in bacteria. Remarkably, except for the mechanisms of DNA synthesis themselves (Marraccino et al, pp 262-268), there is as yet no evident overlap with the mechanisms being elucidated in eukaryotic cells.

The conservation of fundamental mechanisms has clearly greatly accelerated research by allowing particular experiments to be done in the most convenient available systems, and then extrapolated instantly to other systems. However, it is worth noting that there is some danger of carrying this approach too far. For example, it seems clear that the role of Saccharomyces cerevisae Ras proteins in controlling adenylate cyclase is not duplicated in mammalian systems (Cooper, pp 285-295), a result that still seems surprising given the host of other similarities between the yeast and mammalian Ras systems (e.g. Ballester et al, Cell 1989, 59:681-686; Tanaka et al, Cell $1990,60: 803-807)$. In this regard, it will be most interesting to see whether mammalian homologues of the $\mathrm{pu}$ -

Abbreviations

MPF - maturation-promoting factor; PCR-polymerase chain reaction. 
tative guanine nucleotide exchange factors for yeast Ras proteins (Boy-Marcotte et al, Gene 1989, 77:21-30; Powers et ah, Mol Cell Biol 1989, 9:390-395; Hughes et al., Nature 1990, 344:355-357) will fill the present gap in the analogy between mammalian Ras proteins and receptorlinked G-proteins (Cooper, pp 285-295). It should also be noted that it remains unclear whether all 'cyclins' really do cycle in protein abundance (see below), or whether the periodic accumulation of string mRNA (Glover, pp 258-261) has a parallel in other systems that involve a cdc25+-like protein (Futcher, pp 246-251; Morris, pp 252-257).

With these thoughts in mind, it is easy to cheer the continuing study of cell-cycle mechanisms in a wide variety of organisms (Berger, Curr Opin Cell Biol 1989, 1:256-262; Berger and Ching, Exp Cell Res 1989, 182:90-104; Wright and Tollon, J Cell Pbysiol 1989, 139:346-353). Such studies should provide critical tests of the supposed ubiquity of common mechanisms as well as (perhaps) revealing some novel and interestingly different ways of achieving common biological ends.

\section{The mechanics of cellular reproduction}

The past year has seen considerable progress in the elucidation of how a cell reproduces, once it has decided to do so. For example, consider the central event of DNA replication. Although Watson and Crick explained the general principle of this process in their original 1953 paper, it has been rather a long slog to work out the details. Marraccino et al. ( $p p$ 262-268) make clear that the pace has now picked up dramatically. Similarly, exciting progress is being made in understanding the nature and function of centrosomes, kinetochores, and spindle microtubules in eukaryotes (Cande, pp 301-305; McIntosh and Koonce, Science 1989, 246:622-628), the temporal and spatial control of septation in prokaryotes (Lutkenhaus, pp 241-245), and the checkpoints and other coor dinating mechanisms that help tie the individual events of the cell cycle together into a coherent whole (Morris, pp 252-258). These coordinating mechanisms appear to exploit regulation at all possible levels, from control of transcription to control of protein degradation (see espe cially Johnston, pp 274-279, but also most of the other reviews that follow). Particularly striking to me is the pervasiveness of post-translational control, including the apparent regulation of protein activity by controlling complex formation or intracellular localization (Futcher, pp 246-251; Johnston, pp 274-279; Cooper, pp 285-295); I anticipate the discovery of many more examples of control at these levels.

\section{The control of cellular reproduction}

The past year has seen a continuing intense and very suc. cessful focus on mitotic control by the $\mathrm{Cdc} 2$ /maturation- promoting factor (MPF) system (Futcher, pp 246-251; Morris, pp 252-257; Doree, pp 269-273). While joining in the general euphoria, I would also like to note three caveats. First, I remain unconvinced that all of the proteins now referred to as 'cyclins' really do cycle in abundance as do the original proteins of this name. In particular, the case for Schizosaccharomyces pombe $\mathrm{p} 56^{\mathrm{cdcl} 3}$ needs strengthening. The periodic appearance of this protein in the nucleus (Booher et al, Cell 1989, 58:485-497) is a fascinating observation, but does not necessarily reflect periodic changes in protein abundance, as the parallel studies of p34cdc2 clearly show. The dramatic changes in $\mathrm{p} 56 \mathrm{cdcl} 3$ abundance after release from a cdc25 block (Booher et al., 1989; Moreno et al., Cell 1989, 58:361-372) are suggestive, but not conclusive, as such mechanisms of synchronization have produced many seductive artifacts in the past (Creanor $e t$ al., J Cell Sci 1983, 61:339-349). Second, it seems unlikely to me that all of the proteins now being reported to be substrates for $\mathrm{Cdc} 2 / \mathrm{MPF}$ phosphorylation (Doree, pp 269-273) really are meaningful substrates in vivo. It is well to remember the long struggle, only now, perhaps, being rewarded (Cooper, pp 285-295) to identify meaningful substrates for the tyrosine-phosphorylating kinases of animal cells. Third, as our reviewers dealing with mammalian cell proliferation remind us (Zetterberg, pp 296-300; Lau, pp 280-284; Cooper, pp 285-295), understanding of the overall control of cell growth and proliferation will presumably require more attention to the $\mathrm{G}_{1}$ role of $\mathrm{Cdc} 2 / \mathrm{Cdc} 28$ and their homologues. Although exciting progress in this area is being made in studies of S. cerevisiae (Futcher, pp 246-251; Richardson et al., Cell $1989,59: 1127-1133$ ), there is as yet little counterpart in studies of other cell types. Moreover, even in the $S$. cere visiae studies there are still many gaps; for example, there is as yet no published evidence that the putative ' $G_{1} \mathrm{cy}$ clins' really do cycle in abundance.

\section{Conclusions}

In the excitement of the progress being made in the cellcycle field, it is easy to lose track of how far we still have to go. I think that careful reading of the reviews that follow will alleviate any such symptoms. Although Zetterberg (pp 296-300), Lau (pp 280-284), and Cooper (pp 285-295) have done a outstanding job of extracting or der from a vast and (to me, at least) confusing literature, it is hard to come away from their reviews with the feeling that a complete understanding of the control of mammalian cell proliferation is just around the corner. I have the same reaction to the $\mathrm{Cdc} 2 / \mathrm{MPF}$ story, with its current plethora of possible substrates, activators, inhibitors, and protein-protein interactions, as well as to many other topics covered in the reviews that follow. Some may regard this as a pessimistic view, but that is not my mood. Rather, I feel optimistic that we will still have deep and fascinat ing mysteries to marvel at (and study) for many years to come. 